\title{
Novel mouse mammary cell lines for in vivo bioluminescence imaging (BLI) of bone metastasis
}

\author{
Celeste Bolin ${ }^{1}$, Caleb Sutherland ${ }^{1,2}$, Ken Tawara ${ }^{1}$, Jim Moselhy ${ }^{1}$ and Cheryl L. Jorcyk ${ }^{1 *}$
}

\begin{abstract}
Background: Tumor cell lines that can be tracked in vivo during tumorigenesis and metastasis provide vital tools for studying the specific cellular mechanisms that mediate these processes as well as investigating therapeutic targets to inhibit them. The goal of this study was to engineer imageable mouse mammary tumor cell lines with discrete propensities to metastasize to bone in vivo. Two novel luciferase expressing cell lines were developed and characterized for use in the study of breast cancer metastasis to bone in a syngeneic mouse model.

Results: The 4 T1.2 luc3 and 66c14 luc2 cell lines were shown to have high levels of bioluminescence intensity in vitro and in vivo after orthotopic injection into mouse mammary fat pads. The 4 T1.2 luc3 cell line was found to closely model the sites of metastases seen in human patients including lung, liver, and bone. Specifically, 4 T1.2 luc3 cells demonstrated a high incidence of metastasis to spine, with an ex-vivo BLI intensity three orders of magnitude above the commercially available 4 T1 luc2 cells. 66c14 luc2 cells also demonstrated metastasis to spine, which was lower than that of 4 T1.2 luc3 cells but higher than 4 T1 luc2 cells, in addition to previously unreported metastases in the liver. High osteolytic activity of the 4 T1.2 luc3 cells in vivo in the bone microenvironment was also detected.
\end{abstract}

Conclusions: The engineered 4 T1.2 luc3 and 66c14 luc2 cell lines described in this study are valuable tools for studying the cellular events moderating the metastasis of breast tumor cells to bone.

Keywords: Breast cancer, Mammary cancer, Bone metastasis, in vivo imaging, 4 T1 cells, 4 T1.2 cells, Osteolysis, Syngeneic Balb/c model

\section{Background}

Metastasis of breast carcinoma cells from the primary tumor to secondary organ sites such as lung, liver, brain, and bone is the leading cause of mortality in patients with breast cancer [1]. Bone is the most frequent site of metastasis in breast cancer patients, observed in approximately 65 to $75 \%$ of patients with metastatic breast cancer [2]. Bone metastases often cause significant pain and morbidity in these patients due to osteolysis and bone resorption, and the median survival time after detection these metastases is approximately two years $[3,4]$.

Researchers studying breast tumorigenesis and metastatic progression in vivo utilize several types of mouse models including transgenic, xenograft, and syngeneic mouse models. Transgenic mouse models that generate

\footnotetext{
* Correspondence: cjorcyk@boisestate.edu

'Department of Biological Sciences, Boise State University, Boise, ID, USA

Full list of author information is available at the end of the article
}

spontaneous mammary tumors have been developed using promoters such as the mouse mammary tumor virus (MMTV) promoter to drive oncogenes, including polyoma middle T antigen (MMTV-PyMT) and ErbB2/ Neu (MMTV-Neu) (for review see [5]). Transgenic mice lacking the $\mathrm{p} 53$ tumor suppressor gene $\left(\mathrm{p} 53^{-/-}\right)$, which is mutated in $40-50 \%$ of human breast cancers, have also been utilized extensively in cancer studies but do not reproducibly form mammary tumors [6]. A major drawback to these transgenic models, along with the commonly used C3(1)-SV40 T-antigen transgenic mice that also develop mammary tumors independent of steroid supplementation, is that bone metastases cannot be detected (for review see [7]). Given that these tumors occur spontaneously via transformation of the host's normal cells that do not have specific, imageable cellular tags, it is very difficult to track these cells in vivo during tumor progression and metastasis.

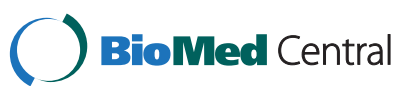


In the xenograft mouse model, human breast cancer cells are injected into immunocompromised, athymic mice. This model is useful because it allows breast cancer cells of human origin to be studied. However, when studying the metastatic cascade in these models, in which the host organism and the implanted tumor cells are not of the same species, important tumor and host stromal interactions may be disrupted due to interspecies signaling incompatibilities [8]. Additionally, due to the compromised immune system of the athymic mouse, immune-mediated tumor and host stromal interactions important for metastasis may be lost in this model (for review see [9]).

Syngeneic models in which the tumor cells are placed into the same species they originated from, and therefore the tumor microenvironment is of the same species, are able to overcome limitations of both xenograft and transgenic models in studying metastasis. Commonly used syngeneic mouse models of breast cancer utilize cells isolated from mammary tumors that occurred spontaneously in wild-type Balb/c mice. These cells can then be injected orthtopically into Balb/c mice and will reproducibly grow a mammary tumor. Currently, there is a series of mouse mammary cancer cell lines derived from the spontaneous breast tumors of Balb/c mice including non-metastatic 67NR cells, 66c14 cells that metastasize to lung, and $4 \mathrm{~T} 1$ cells that metastasize to lung and liver [10]. 4 T1.2 cells are a sub-clone of the original osteolytic $4 \mathrm{~T} 1$ mammary tumor cell line that have been selected for their increased propensity to metastasize to bone [10]. While $66 \mathrm{c} 14$ cell metastasis is restricted to the lymph nodes and lung, 4 T1.2 cells closely resemble the metastatic profile in humans with metastasis to the bone, lymph nodes, and lung [10,11]. Additionally, the $4 \mathrm{~T} 1.2$ orthotopic model results in an increase in hypercalcemia due to osteolysis, an important characteristic that resembles human bone metastasis [12]. Engineering these mouse cells to be traceable and imageable in a live animal make them even more valuable for studying metastasis to bone in vivo in a syngeneic mouse model.

Currently, there are several human breast cancer cell lines engineered to express bioluminescence imaging (BLI) tags such as luciferase (luc) that can be used to track the metastases of these cells in vivo. These cell lines include MDA-MB-231-luc2 and MCF-7-luc2, both available commercially (Caliper Life Sciences, Hopkinton, MA). In general, human mammary cancer cells utilized in xenograft mouse models of breast cancer demonstrate a low propensity to metastasize to bone unless introduced via intra-cardiac injection, which bypasses critical initial steps in the metastatic cascade (for review see [13]). To date, there are only two mouse mammary cancer cell lines commercially available that stably express the luc gene, 4 T1-luc2 and 4 T1-luc2-GFP cells, available from Caliper Life Sciences (Hopkinton, MA, USA).

In this study we generated by transfection of $66 \mathrm{c} 14$ and 4 T1.2 mouse mammary carcinoma cells with the pGL4 vector two new cell lines that stably express the luciferase gene. These two cell lines, referred to as $66 \mathrm{c} 14$ luc2 and 4 T1.2 luc3, produce high levels of bioluminescence in vitro and in vivo. Additionally, tumor progression in vivo was consistent with the parental 66c14 and 4 T1.2 cell lines. Importantly, the overall metastatic burden, quantified as the number and photon density of BLI areas, was higher in mice injected orthotopically with the 4 T1.2 luc3 cells as compared to both $66 \mathrm{c} 14$ luc 2 cells and the commercially available 4 T1 luc2 cells (Caliper Life Sciences, Hopkinton, MA). In vivo and ex vivo imaging of metastasis showed that the $4 \mathrm{~T} 1.2$ luc3 cells had a statistically significant higher level of metastases to the spine and lung as compared to the $66 \mathrm{c} 14$ luc2 cells, which nonetheless showed detectable levels of spine and lung metastases. Therefore, the newly established 4 T1.2 luc3 and 66c14 luc2 cell lines are reliable tools for monitoring and quantifying breast cancer metastasis to bone and other organs as well as for studying the signaling pathways associated with these processes.

\section{Results}

Establishment and characterization of $66 \mathrm{c} 14$ and 4 T1.2 mouse mammary carcinoma cell lines that stably express luciferase

Of the five different 66c14 luc lines produced, 66c14 luc5 cells produced the highest bioluminescence intensity, 1,019 photons/sec/cell, and the $66 \mathrm{c} 14$ luc 2 cells produced the second highest, 945 photons/sec/cell (Figure 1A, green bar). Of the five different 4 T1.2 luc lines produced, 4 T1.2 luc3 cells produced the highest level of bioluminescence intensity, at 2,070 photons/sec/ cell (Figure 1B, green bar). Two cell lines, 66c14 luc1 (105 photons/sec/cell) and 4 T1.2 luc5 (150 photons/ sec/cell), had bioluminescence intensities below the minimum detection limit (MDL) for in vivo imaging ( 300 photos/sec/cell; Caliper Life Sciences) and were not utilized in the following in vivo studies. The other eight luc-expressing cell lines generated in 4 T1.2 and $66 \mathrm{c} 14$ cells were further assessed for in vivo BLI based on in vitro bioluminescent intensities at least twice the in vivo MDL.

\section{In vivo tumor growth of $66 \mathrm{c} 14$ luc2 and 4 T1.2 luc3 cells}

To confirm that the luc-expressing cell lines grow in vivo in a manner comparable to the parental cells, tumor growth was monitored. $1 \times 10^{5}$ cells (parental 66c14, $66 \mathrm{c} 14$ luc2, 66c14 luc3, 66c14 luc4, and 66c14 luc5 or 

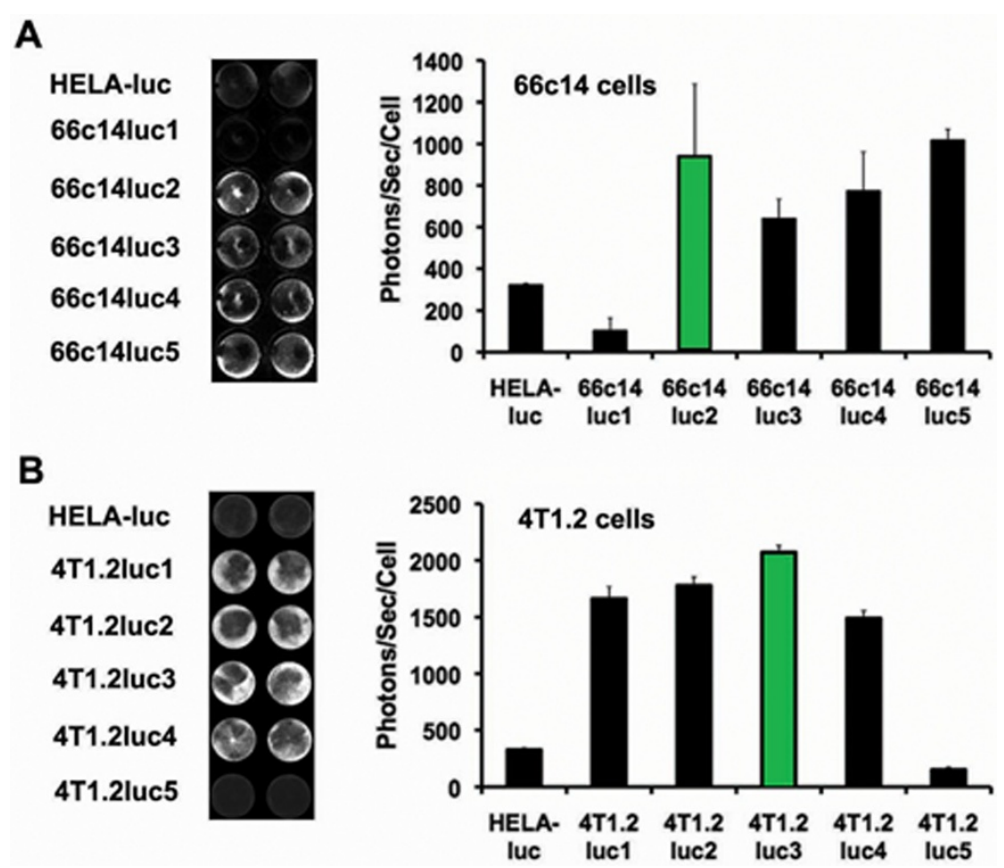

Figure 1 Bioluminescence intensities of $66 \mathrm{c} 14$ luc- and 4 T1.2 luc-expressing cell lines in vitro. Image analysis show bioluminescence from clonal populations of (A) 66c14 luc(1-5) cells and (B) 4 T1.2 luc(1-5) cells. Green bars highlight 4 T1.2 luc3 and 66c14 luc2 clones with high mean bioluminescence values. Data is expressed as photons/sec/cell (mean \pm std dev, $n=4$ ) calculated by comparing each cell line to known values of HeLa-Luc cells (350 photons/sec/cell; see Materials and Methods).

parental 4 T1.2, 4 T1.2 luc1, 4 T1.2 luc2, 4 T1.2 luc3, and 4 T1.2 luc4) were injected into the mammary fat pad of female Balb/c mice ( $n=3$ per cell line) (Figure 2). Tumor size was measured using calipers twice a week for up to 36 days and expressed as tumor volume $\left(\mathrm{mm}^{3}\right)=($ length $\times$ width $^{2} / 2$ ) [11]. Mice injected with 66c14 luc2 cells (Figure 2A; green) displayed a tumor growth pattern similar to that of mice injected with parental $66 \mathrm{c} 14$ cells (Figure 2A; red). In vivo analysis of $66 \mathrm{c} 14$ luc3 cells demonstrated a small reduction in maximum tumor volume compared to parental $66 \mathrm{c} 14$ cells but did not produce bioluminescence in vivo (Figure 2A, Figure 3). Mice injected with $66 \mathrm{c} 14$ luc4 or luc5 cells (the cells with high BLI intensities in vitro) had severely reduced tumor growth in vivo (Figure 2A, Figure 3). Injection of $4 \mathrm{~T} 1.2$ luc3 cells (the cells with the highest BLI intensity in vitro; Figure 2B; green) resulted in a tumor growth pattern similar to that of parental 4 T1.2 cells (Figure 2B; red). 4 T1.2 luc1, luc2, and luc4 cell injections all resulted in reduced tumor progression in vivo (Figure 2B, Figure 3). Overall, 66c14 luc2 and 4 T1.2 luc3 cell lines demonstrated tumor progression most consistent with parental cell lines.

Next, BLI intensity of $66 \mathrm{c} 14$ luc and 4 T1.2 luc cells during tumor growth was assessed in vivo. Tumors from injected 66c14 luc2 cells produced the highest level of BLI intensity of all the $66 \mathrm{c} 14$ luc cells, averaging
$8.29 \times 10^{9}$ photons/second on day 25 after tumor cell injection (Figure 4A; green, Figure 3). Tumors from injected 4 T1.2 luc3 cells produced the highest level of BLI intensity of all the 4 T1.2 luc cells, averaging $4.84 \times 10^{6}$ photons/second on day 25 after tumor cell injection (Figure 4B; green, Figure 3). Sequential images on days $18,25,32$, and 39 of mice injected with the $66 \mathrm{c} 14$ luc2 cell line showed increasing BLI intensity at the tumor site correlating with increasing tumor size (Figure 4C). The BLI intensity of tumors from mice injected with 4 T1.2 luc3 cells started to decrease after day 25, likely due to the tumor-associated necrosis (Figure 4D).

\section{BLI of metastases from mice injected with $66 \mathrm{c} 14$ luc2 or} 4 T1.2 luc3 cells

The 66c14 luc2 and 4 T1.2 luc3 cell lines were chosen for additional investigation of metastatic potential due to their consistently similar tumor growth profiles with the parental cell lines, and high levels of BLI intensity in vivo. These two new cell lines were compared to the 4. T1 luc2 cell line purchased from Caliper Life Sciences described previously [14]. Independent orthotopic injection of each of these three cell lines $\left(1 \times 10^{5}\right.$ cells $)$ into the mammary fat pad of female Balb/c mice $(n=3)$ resulted in palpable tumors at day 14 after injection. 


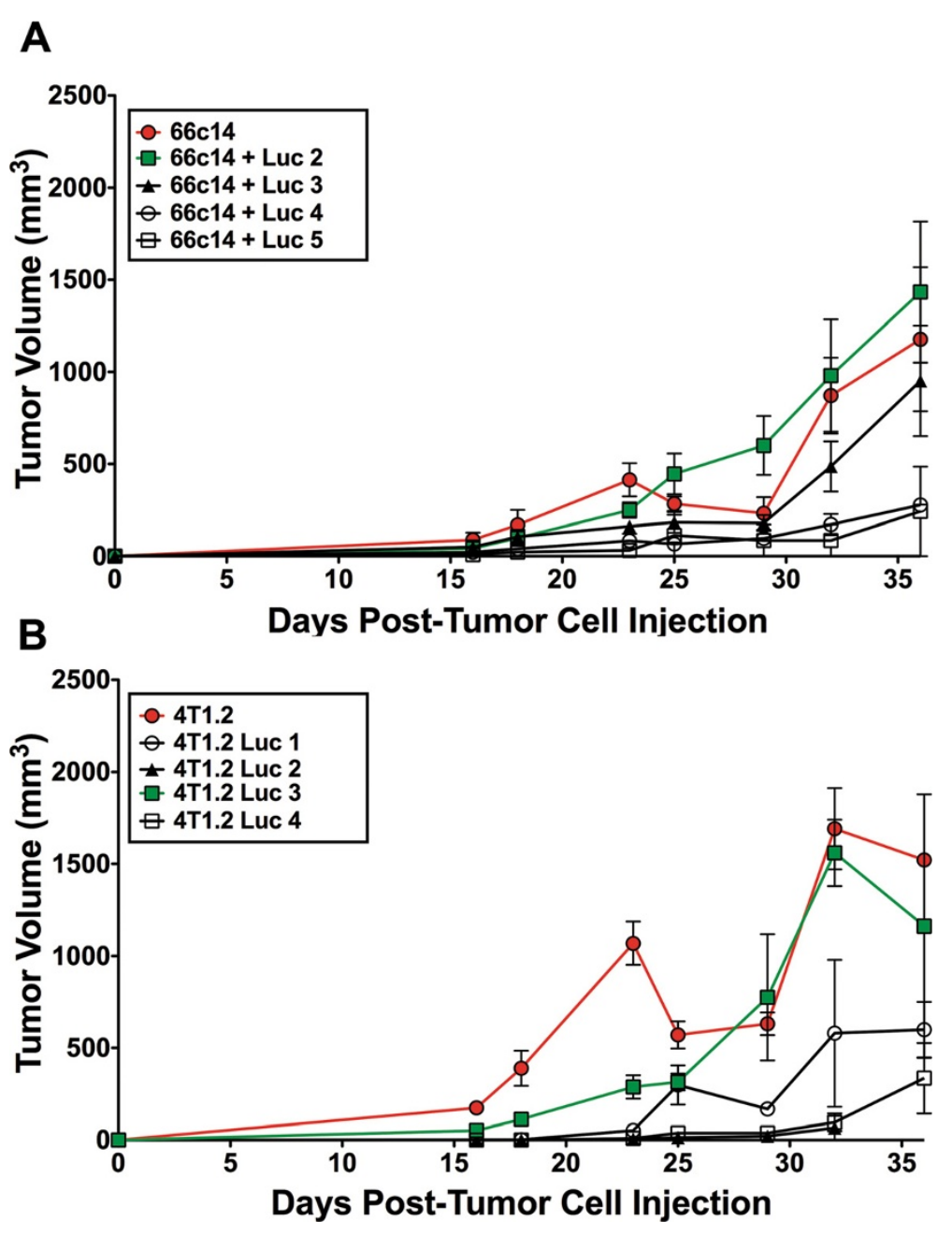

Figure 2 Tumor growth of $66 \mathrm{c} 14$ luc and 4 T1.2 luc cells injected orthotopically into Balb/c mice. $66 \mathrm{c} 14$ luc and 4 T1.2 luc cell lines were injected into the mammary fat pad of Balb/c mice and tumor volume was measured over 36 days. (A) 66c14 luc2 tumor growth (green/square) was most consistent with parental 66c14Tumor growth (red/circle) and (B) 4 T1.2 luc3 tumor growth (green/square) was most consistent with parental 4 T1.2 (red/circle) tumor growth. Data is expressed as tumor volume $\left(\mathrm{mm}^{3}\right)$ (mean \pm std dev; $\left.n=3\right)$.

Mice injected with the 66c14 luc2, 4 T1.2 luc3, or 4 T1 luc 2 cells were imaged once the mice had reached endpoint criteria of maximum metastases including a loss of activity and $10 \%$ of their body weight (32 days after injection for the 66c14 luc2 and 4 T1.2 luc3 injected mice and 28 days for the $4 \mathrm{~T} 1$ luc2 injected mice).

In the dorsal and ventral whole body BLI images, the $66 \mathrm{c} 14$ luc2 and 4 T1.2 luc3 cells were visible in the primary tumors and numerous secondary metastatic regions in vivo using a BLI intensity imaging scale on the order of $10^{6}$ photons $/ \mathrm{sec} / \mathrm{cm}^{2}$ (highlighted with a yellow box in Figure 5A). On the other hand, in whole body BLI images of mice injected with the commercially available 4 T1 luc2 cells only primary tumors were visible. The high bioluminescence produced by the $4 \mathrm{~T} 1$ luc2 primary tumor was imaged using a scale on the order of $10^{8}$ photons $/ \mathrm{sec} / \mathrm{cm}^{2}$ (highlighted with a red box in Figure 5A), and likely prohibited secondary sites of metastases to be visualized.

Ex vivo analyses were also performed in order to identify specific tissues where metastases formed. BLI revealed 66c14 luc2 ex vivo metastases primarily to lung, although metastases to liver and spine were detectable, while 4 T1.2 luc3 cells were highly metastatic to lung, liver and spine (Figure 5B, Figure 3). Mice injected with the commercially available 4 T1 luc2 cells showed weak ex vivo BLI intensities from metastases in the lung, liver, and spleen, and no spine metastases were measured using the same scale as with the 66c14 luc2 and 4 T1.2 luc3 cells (Figure 5B, yellow box; Figure 3). 


\begin{tabular}{|c|c|c|c|c|c|}
\hline Cell line & $\begin{array}{l}\text { In vitro intensity } \\
\text { (photons/sec/cell) }\end{array}$ & $\begin{array}{l}\text { In vivo intensity } \\
\text { (photons/sec) }\end{array}$ & $\begin{array}{c}\text { Maximum Mean } \\
\text { Tumor Volume } \\
\left(\mathrm{mm}^{3}\right)\end{array}$ & $\begin{array}{l}\text { Metastatic Sites } \\
\text { other than Bone }\end{array}$ & $\begin{array}{c}\text { Number of Animals with Spine } \\
\text { Metastases } \\
\left(>1.0 \times 10^{4} \text { photons } / \mathrm{sec} / \mathrm{cm}^{2}\right)\end{array}$ \\
\hline $66 c 14$ & $\mathrm{~N} / \mathrm{A}$ & $\mathrm{N} / \mathrm{A}$ & 1177.25 & $\begin{array}{l}\text { Lung, Lymph } \\
\text { Nodes }^{b}\end{array}$ & \\
\hline $66 c 14$ luc1* & 106 & $\mathrm{~N} / \mathrm{A}$ & $\mathrm{N} / \mathrm{A}$ & N/A & \\
\hline $66 c 14$ luc2 & 945 & $8.29 \times 10^{9}$ & 1433.33 & $\begin{array}{l}\text { Lung }(3 / 3) \text {, } \\
\text { Liver }(1 / 3) \\
\end{array}$ & $3 / 3$ \\
\hline $66 c 14$ luc3 & 641 & 0 & 952.00 & $\mathrm{~N} / \mathrm{A}$ & \\
\hline $66 c 14$ luc4 & 775 & $5.27 \times 10^{9}$ & 277.33 & $\mathrm{~N} / \mathrm{A}$ & \\
\hline $66 c 14$ luc5 & 1,019 & $4.96 \times 10^{9}$ & 243.00 & $\mathrm{~N} / \mathrm{A}$ & \\
\hline $4 \mathrm{~T} 1.2$ & $\mathrm{~N} / \mathrm{A}$ & $\mathrm{N} / \mathrm{A}$ & 1691.25 & $\begin{array}{c}\text { Lung, Lymph } \\
\text { Nodes, Brain, Bone }\end{array}$ & \\
\hline 4T1.2 luc1 & 1,661 & $3.91 \times 10^{4}$ & 599.00 & $\mathrm{~N} / \mathrm{A}$ & \\
\hline 4T1.2 luc2 & 1,778 & $2.16 \times 10^{4}$ & 65.33 & $\mathrm{~N} / \mathrm{A}$ & \\
\hline 4T1.2 luc3 & 2,072 & $4.84 \times 10^{6}$ & 1560.00 & $\begin{array}{l}\text { Lung }(3 / 3) \text {, } \\
\text { Liver }(3 / 3)\end{array}$ & $3 / 3$ \\
\hline 4T1.2 luc4 & 1,491 & $6.41 \times 10^{4}$ & 335.25 & $\mathrm{~N} / \mathrm{A}$ & \\
\hline 4T1.2 luc5* & 149 & $\mathrm{~N} / \mathrm{A}$ & $N / A$ & $\mathrm{~N} / \mathrm{A}$ & \\
\hline $\begin{array}{c}4 \mathrm{~T} 1 \text { luc2 } \\
\text { (Caliper Life Sciences) }\end{array}$ & $\sim 6500^{\mathrm{a}}$ & $6.66 \times 10^{9}$ & 1389.00 & $\begin{array}{l}\text { Lung (4/4), Liver } \\
\text { (4/4), Spleen (3/4) }\end{array}$ & $0 / 4$ \\
\hline $\begin{array}{l}\text { * Indictes cells were } \\
\text { aCCaliper Life Scences, } \\
\text { 'Lelekakis, M., oseley, J } \\
\text { Experimental Metastasi }\end{array}$ & $\begin{array}{l}\text { not injected into mice } \\
\text { ppkinton, MA, USA } \\
\text { Martin, T., Hards, D., Will } \\
\text { 163-170 }\end{array}$ & $\begin{array}{l}\text { lue to low in vitro lur } \\
\text { ims, E., Ho, P., Lowen, }\end{array}$ & $\begin{array}{l}\text { inescence. } \\
\text {., Javni, J., Miller, F., S }\end{array}$ & lavin, J., and Anderson, R. & (1999) Clinical \& \\
\hline
\end{tabular}

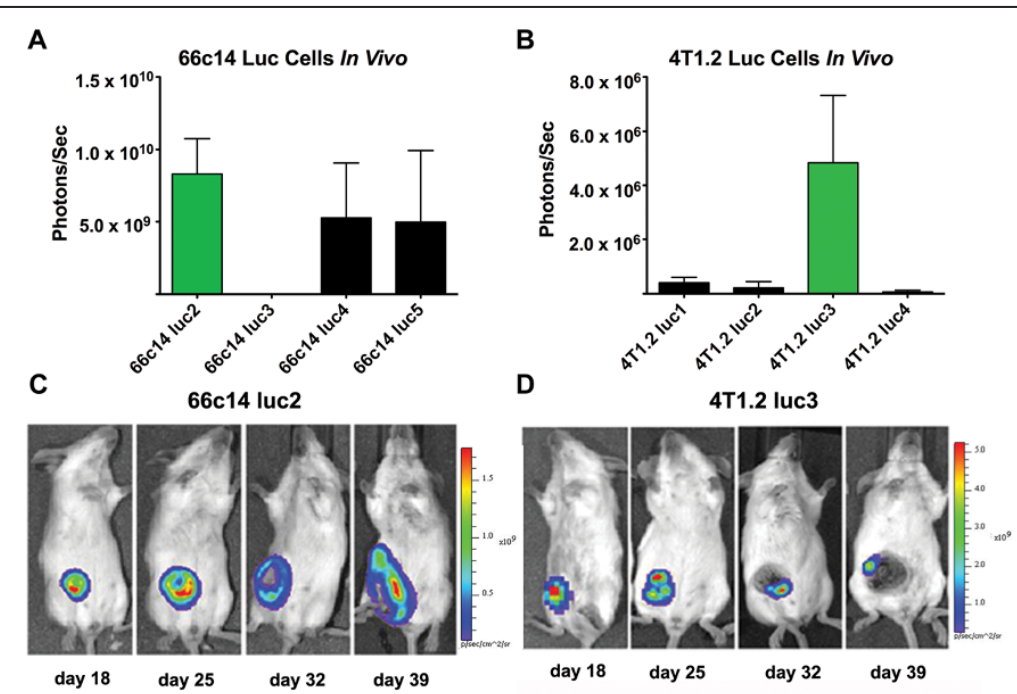

Figure $4 \mathrm{BLI}$ of 66c14 luc and 4T1.2 luc cells in vivo. Tumor bioluminescence intensities from each of the (A) $66 \mathrm{c} 14$ luc and (B) 4 T1.2 luc tumor-bearing mice on day 25 after tumor cell injection are shown. 66c14 luc2 and 4 T1.2 luc3 exhibited the highest BLI intensity in vivo (green bars). Sequential images of (C) 66c14 luc2 and (D) 4 T1.2 luc3 tumor-bearing mice imaged ventrally by BLI once a week for 4 weeks are shown. 66c14 luc2 tumors continued to grow while 4 T1.2 luc3 tumors became necrotic between day 25 and 32. Data expressed as photon/sec (mean \pm std dev; $n=3)$. 

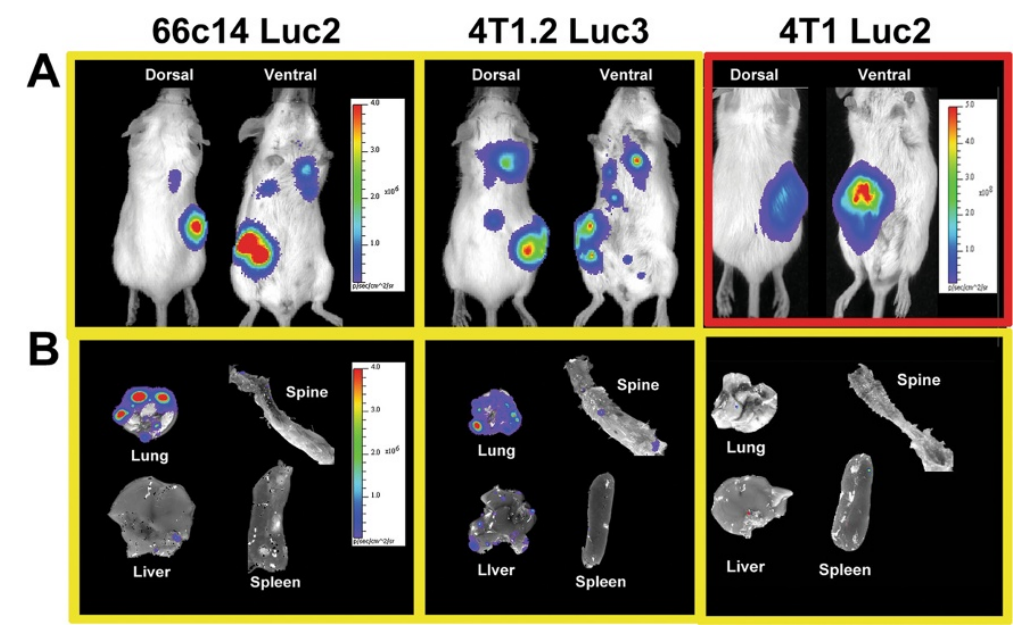

Figure 5 Metastasis of $66 \mathrm{c} 14$ luc2, 4 T1.2 luc3, and 4 T1 luc2 cells in vivo. Representative (A) whole animal and (B) ex vivo organs from mice injected with the 66c14 luc2 (left), 4 T1.2 luc3 (middle), or commercially available 4 T1 luc2 (right) cells, 4-5 weeks after injection are shown. Primary tumor bioluminescence intensities were visualized in animals injected with all cell types in both dorsal and ventral views. Bioluminescence was also detectable in the thoracic region of the 66c14 luc2 and 4 T1.2 luc3 animals correlating with ex vivo bioluminescence from metastatic lesions detected in lung and spine. No bioluminescence representing metastatic lesions was detected in mice injected with 4 T1 luc2 cells. The bioluminescence intensity of the primary tumor from the $4 \mathrm{~T} 1$ luc2 mouse represented was very high and likely prohibited secondary sites of metastasis from being visualized. The BLI scale next to the whole body image of the $4 \mathrm{~T} 1$ luc2 injected mouse (highlighted in red) reflects this difference. All other images (highlighted in yellow) are presented with a consistent BLI scale. Overall, the 4 T1.2 luc3 cells showed the highest levels of BLI from metastases both in vivo and ex-vivo.

Quantification of the BLI intensities from spines, lung, and liver ex vivo from mice injected with 66c14 luc2, 4. T1.2 luc3, and 4 T1 luc2 cells were performed. Results showed that spines excised from the 4 T1.2 luc3 group had BLI intensities 10-fold higher than the 66c14 luc2 group and three orders of magnitude $\left(10^{3}\right)$ higher than that of the $4 \mathrm{~T} 1$ luc2 injected group (Figure 6A). Lungs from mice injected with either 66c14 luc2 or 4 T1.2 luc3 cells had a mean BLI intensity an order of magnitude higher than the $4 \mathrm{~T} 1$ luc2 (Figure $6 \mathrm{~B}$ ). Interestingly, one of the mice injected with $66 \mathrm{c} 14$ luc2 cells also showed a high average BLI intensity from multiple metastases in the liver, never previously reported in the parental 66c14 cell line (Figure 6C). Overall, the metastatic burden, which accounts for the average number and size of metastases, detected in vivo and ex vivo was highest for 4 T1.2 luc3 cells. In terms of their ability to be tracked in the primary tumor and secondary metastatic sites

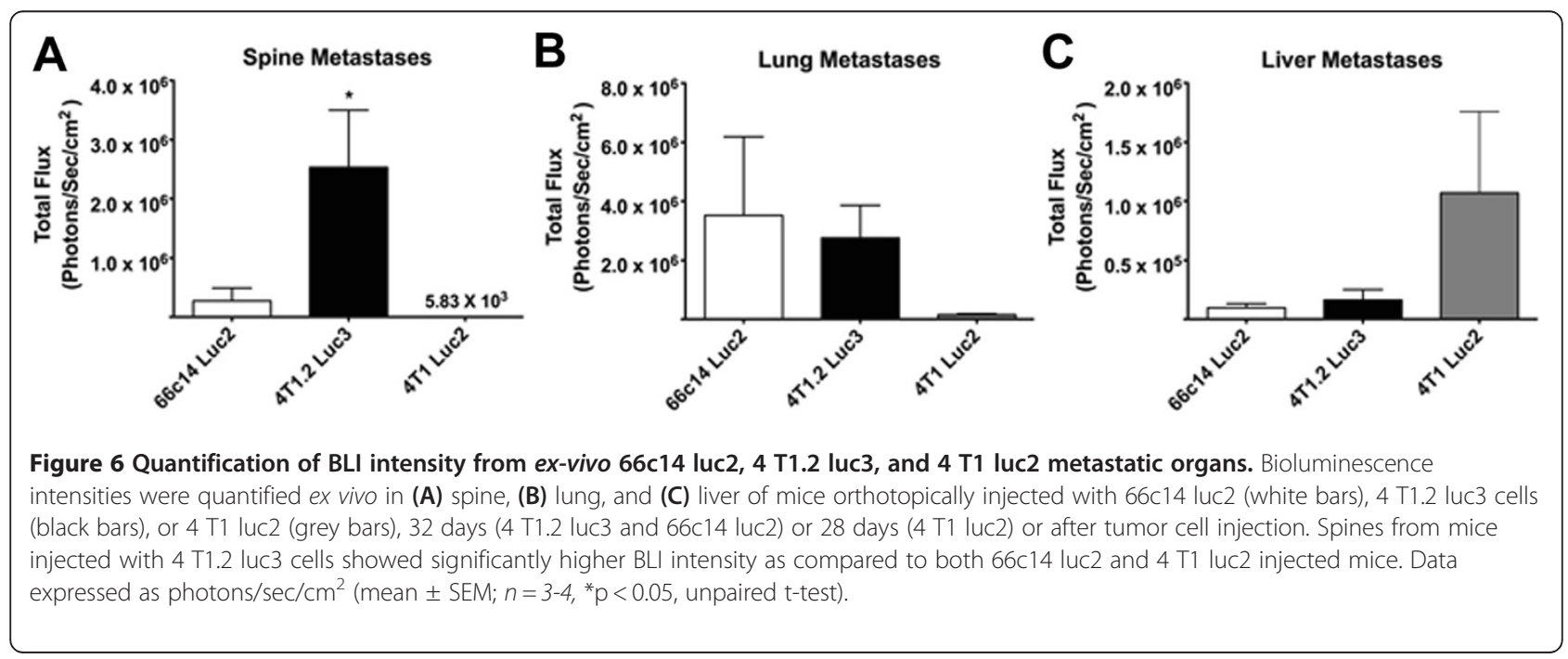


in vivo and ex vivo, both 4 T1.2 luc3 and 66c14 luc2 cells showed higher BLI intensities than 4 T1 luc2 cells.

\section{Micro-computed tomography (Micro-CT) analysis of 4 T1.2 luc3 cells}

To identify whether 4 T1.2 luc3 cells are osteolytic by comparison to the commercially available 4 T1 luc2 cells, each of the cell lines were injected into the tibia of 6-week old female Balb/c mice and analyzed for bone integrity using micro-CT. Whole body and ex vivo BLI analysis of mice injected with 4 T1.2 luc3 and 4 T1 luc2 cells verified the presence of tumor cells 18 days after injection into the tibia (Figure 7A). Micro-CT analysis showed significant osteolytic activity of both cell lines near the injection site at the proximal tibia (Figure 7B-i) and normal bone at the distal tibia (Figure 7B-ii). 66c14 luc2 cells were not analyzed for osteolytic potential in this study. Overall, these results suggest that 4 T1.2 luc3 cells are a useful model for studying osteolytic bone metastasis originating from a primary breast tumor.

\section{Discussion}

This study presents two new luciferase-expressing mouse cell lines (66c14 luc2 and 4 T1.2 luc3 cells) useful for the in vivo study of breast cancer metastasis to bone. Using BLI, these cell lines demonstrate greater bone

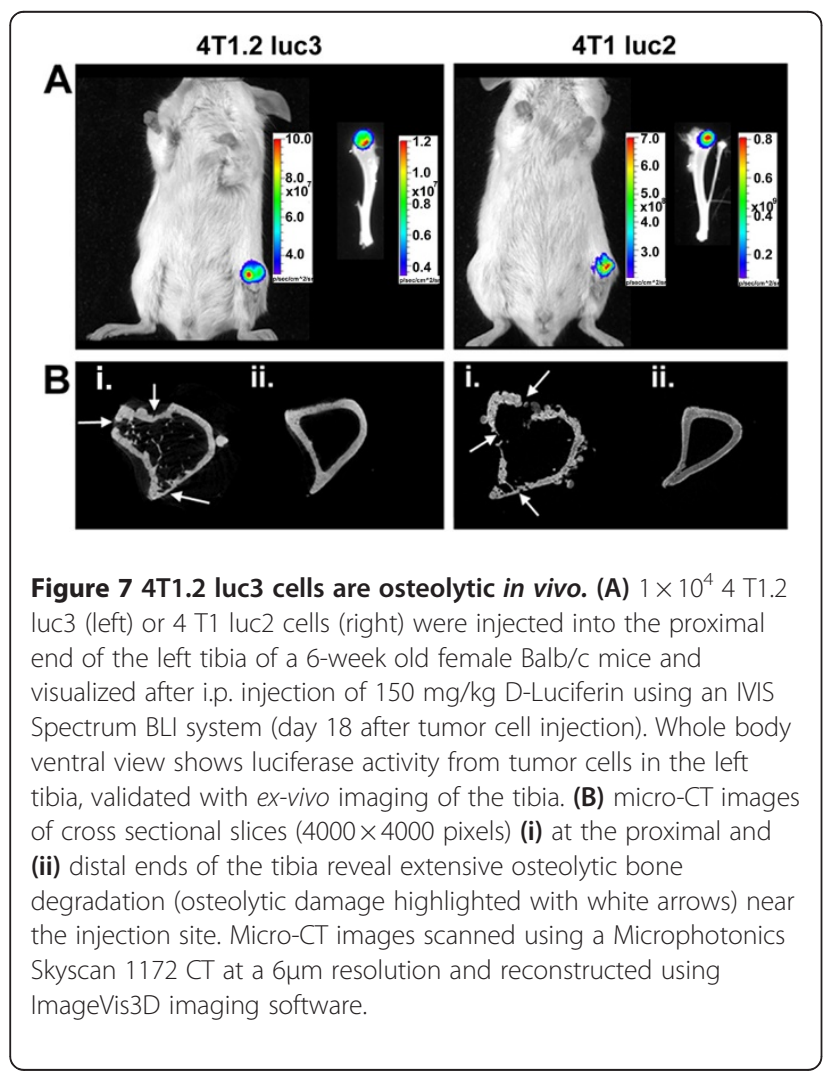

metastatic potential in a syngeneic Balb/c mouse model than the commercially available 4 T1 luc2 cell line (Caliper Life Sciences, Hopkinton, MA). The 4 T1.2 luc3 cell line is metastatic to the lung, liver, and bone in $100 \%$ of the mice tested, and the $66 \mathrm{c} 14$ luc 2 cell line is highly metastatic to lung and less metastatic to bone based on BLI intensities. Each of the two cell lines showed in vitro growth rates (data not shown) and in vivo tumor sizes similar to the parental 4 T1.2 and 66c14 cell lines.

Lung and bone are the predominant destinations of metastatic 4 T1.2 cells [10,11]. However, here we report detectable metastases in the liver of mice injected with the 4. T1.2 luc3 cell line as well as the 66c14 luc2 cell line, a site of metastases never previously identified with the parental 4 T1.2 or $66 \mathrm{c} 14$ cells although demonstrated with the 4 T1 cells [10]. It is possible that these cells have increased metastatic potential due to incorporation of the luciferase gene and/or due to the transfection and clone selection process. This increased metastatic potential displayed by the luciferase-expressing cell lines is further supported by our ability to detect low levels of bioluminescence from the spines of mice treated with the $66 \mathrm{c} 14$ luc 2 cells, when previous studies have not shown detectable metastases to bone using the parental 66c14 cell line [10]. However, it is important to note that the bioluminescence intensity from metastatic 4 T1.2 luc3 cells in the spine was nearly ten-fold greater than that of the $66 \mathrm{c} 14$ luc 2 cells and three orders of magnitude greater $\left(10^{3}\right)$ that that of the 4 T1 luc 2 cells, although the number of metastases detected was similar for all three cell lines (data not shown). This could be partially explained by differences in tumor growth rate and size at the endpoint of the experiment of mice injected with the 4 T1.2 luc3 cells compared to the $66 \mathrm{c} 14$ luc 2 or 4 T1 luc2 cells (data not shown). In general, a slower progression of the primary tumor has been correlated with increased metastasis in mouse models [5].

Orthotopically injected 66c14 luc2 or 4 T1.2 luc3 cells outperformed $4 \mathrm{~T} 1$ luc2 cells in whole-body BLI for the detection of metastases. Bioluminescence was detected in the thoracic and abdominal regions of whole mouse images suggesting that metastasis of 66c14 luc2 and 4 T1.2 luc3 cells had occurred. This was verified by the presence of metastases in the lungs, spine, and liver ex vivo. There was no bioluminescence detected in these regions of the mice injected with the $4 \mathrm{~T} 1$ luc 2 cells using IVIS instrument settings appropriate for visualizing the primary tumor. Although there were verified metastases detected in the lung, spine, and liver by ex vivo imaging from mice injected with the $4 \mathrm{~T} 1$ luc 2 cells, the intensities were several orders of magnitude lower than the primary tumor. It is important to note that the much higher luminescence of the 4 T1 luc2 primary tumor makes bioluminescence visualization and quantification of secondary sites of metastasis in the whole mouse difficult. 
To establish an in vivo model system where the number of metastatic sites was increased by bypassing initial steps of the metastatic cascade, intra-cardiac injections were performed. Injection of $4 \mathrm{~T} 1.2$ luc3 cells into the left ventricle resulted in cell migration to and colonization in lung, liver, spine, brain, spleen, and kidneys (data not shown). The same metastatic sites were seen with 4 T1 luc 2 cells and a similar pattern was seen with 66c14 cells, with the exception of spleen and brain (data not shown). Therefore 4 T1.2 luc3 cells are particularly useful for investigations of mammary tumor metastatic sites including brain and kidney if administered via intra-cardiac injection.

Intra-tibial injections of $4 \mathrm{~T} 1.2$ luc3 and $4 \mathrm{~T} 1$ luc2 cells were performed to evaluate the osteolytic nature of these cells. Parental 4 T1 cells have been extensively characterized in vivo for their osteolytic activity in bone [15-17], while no previous publications address either parental 4 T1.2 or 4 T1 luc2 cells. Our results demonstrated that both 4 T1.2 luc3 and 4 T1 luc2 cells have similar osteolytic activity in vivo (Figure 7 ). Therefore, these cells are useful in BLI and 3-D bone morphology studies performed in tandem investigating not only metastasis, but also the osteolytic capacity of mammary tumor cells.

\section{Conclusions}

The purpose of this study was to incorporate the luciferase gene into previously characterized mouse mammary tumor cell lines with discrete bone metastatic profiles in order to track metastases in vivo using BLI. We show that the highly metastatic 4 T1.2 luc3 cell line, when injected orthotopically, closely models the sites of metastases seen in human patients including lung, liver, and bone. These cells show osteolytic activity in vivo and the ability to proliferate in additional organ sites such as brain, spleen, and kidneys. We also present results demonstrating a phenotypically similar 66c14 luc2 cell line that shows similar metastases in the lung as the 4 T1.2 luc3 cell line along with previously unreported metastases in bone. These imageable cells lines are powerful tools for identifying mechanisms important for breast cancer metastasis to bone in vivo.

\section{Methods}

\section{Cells lines and cell culturing}

$66 \mathrm{c} 14$ and 4 T1.2 cells were cultured and maintained in $\alpha$-MEM supplemented with $10 \%$ fetal bovine serum, $1 \mathrm{mM}$ Penicillin/Streptomycin, and $1 \mathrm{mM}$ sodium pyruvate at $37^{\circ} \mathrm{C}$ in $5 \% \mathrm{CO}_{2}$ and $95 \%$ humidity. $4 \mathrm{~T} 1$ luc2 cells were purchased from Caliper Life Sciences (Hopkinton, MA) and grown according to manufacturer's instructions.
Stable transfections of cells containing the pGL4 vector Approximately $2.5 \times 10^{5} 66 \mathrm{c} 14$ and $4 \mathrm{~T} 1.2$ cells were plated in 6-well culture dishes and transfected with $5.0 \mu \mathrm{g}$ of pGL4.13[luc2/SV40]vector (Promega, Madison, WI) using Lipofectamine LTX (Invitrogen, Carlsbad, CA) per manufacturer's instructions. The cells were trypsinized (0.25\% trypsin) 48 hours after transfection, diluted, and plated into 96-well plates. Two weeks after plating, $100 \mu \mathrm{L}$ media containing D-luciferin (Caliper Life Sciences, Hopkinton, MA) at a final concentration of $150 \mu \mathrm{g} / \mathrm{mL}$ was added to the 96-well plates. Visualization of bioluminescence was performed using the Kodak Image Station 4000R (Eastman Kodak Company, Rochester, NY) with a 5minute exposure. Bioluminescent cells were serially diluted into 96-well plates and cells were grown approximately 2 weeks and assayed for bioluminescence. The colonies with the highest levels of bioluminescence were diluted to 1 cell/100 $\mu \mathrm{L}$ and plated in 96-well plates, grown, and assayed again for bioluminescence. To ensure a clonal population, the colonies with the highest level of bioluminescence were again selected, and the above process was repeated an additional time. Finally, the colonies with the highest level of bioluminescence were selected and expanded to obtain clonal populations of 66c14 luc (66c14 luc1 to $66 \mathrm{c} 14$ luc5) and 4 T1.2 luc (4 T1.2 luc1 to 4 T1.2 luc5) cells expressing the luciferase gene.

\section{In vitro bioluminescence assay to estimate cell line bioluminescence intensities}

Approximately $1 \times 10^{5} 66 \mathrm{c} 14$ luc and $4 \mathrm{~T} 1.2$ luc cells containing stably expressing the pGL4 vector, as well as control HeLa luc cells (Caliper Life Sciences) were plated in a 24-well culture dish and incubated overnight. Bioluminescent intensity was measured as described in the previous section. Using Image J software (National Institutes of Health), each captured image was inverted and total pixel density was calculated. HeLa luc cells, producing approximately 350 photons/sec/cell, were used as a reference point to estimate the photons/sec/ cell produced by each of the new 66c14 luc and $4 \mathrm{~T} 1.2$ luc cell lines.

\section{Injection of cells for measuring in vivo bioluminescence imaging (BLI) and tumor progression}

All animal studies were conducted in accordance with the animal component of research protocol (ACORP) \#JOR0010 protocol approved by the Institutional Animal Care and Use Committee (IACUC) at the Boise VA Medical Center, Boise, ID. $1 \times 10^{5}$ cells suspended in $10 \mu \mathrm{L}$ of phosphate buffered saline (PBS) were injected into the $4^{\text {th }}$ mammary fat pad of 7 -week old female Balb/c mice ( $n=3$ /group). Ten different groups of cells were injected including parental (control) 66c14, 66c14 luc2, 66c14 luc3, 66c14 luc4, 66c14 luc5, parental 
(control) 4 T1.2, 4 T1.2 luc1, 4 T1.2 luc2, 4 T1.2 luc3, and 4 T1.2 luc4 cells. Tumor size was measured with calipers bi-weekly once tumors became palpable (after approximately 2 weeks). BLI of live animals was initiated at 18 days after cell line injection and performed weekly. Briefly, mice were injected i.p. with $150 \mathrm{mg} / \mathrm{kg}$ of D-luciferin (Caliper Life Sciences) in PBS, anesthetized with $2.5 \%$ isoflurane, and imaged. Mice were imaged using a charge-coupled device camera-based bioluminescence imaging system (IVIS Spectrum, Caliper Life Sciences; exposure time 1-300 sec, binning 4/8/16, field of view $23 \mathrm{~cm}, \mathrm{f} / \mathrm{stop} 1$, emission filter open). Signal was measured and recorded as total flux (photons/sec). Corresponding grayscale photographs and color luciferase images were automatically superimposed and analyzed with Living Image software (Xenogen Biosciences Corporation, Cranbury, NJ).

Mice utilized for metastasis investigations were treated exactly as above with the following modifications. Three mice per cell line were orthotopically injected with 66c14 luc2, 4 T1.2 luc3, or 4 T1 luc2 cells. Whole animals were imaged 4-5 weeks following tumor cell injection and immediately injected with a second dose of $150 \mathrm{mg} / \mathrm{kg}$ of D-luciferin. Ten minutes following this injection the mice were euthanized with isoflurane gas followed by cervical dislocation, and the lungs, spine, spleen, and liver were dissected and imaged ex vivo using the bioluminescence imaging system (exposure time 1-300 sec, binning of 4/8/16, field of view $23 \mathrm{~cm}$, $\mathrm{f} /$ stop of 1 , emission filter open).

\section{Micro-computed tomography (Micro-CT)}

In order to verify the osteolytic potential of the $4 \mathrm{~T} 1.2$ luc3 cells, $1 \times 10^{4}$ cells in $100 \mu \mathrm{L}$ of PBS were injected into the left tibia of a 6-week old female Balb/c mouse. In vivo bioluminescence was determined as stated above. Immediately following ex-vivo imaging of the tibia, it was placed in $10 \%$ formalin overnight and stored in $70 \%$ ethanol for analysis. Micro-CT analysis was conducted using a Microphotonics Skyscan 1172. A $6.6 \mu \mathrm{M}$ resolution was used for image collection of $2 \mathrm{D}$ scans $(4000 \times 4000$ pixels $)$ and ImageVis3D imaging software (www.imagevis3d.org) for 3D reconstructions.

\section{Competing interests}

The authors declare that they have no competing interests.

\section{Authors' contributions}

CS was responsible for the in vitro characterization of the luciferaseexpressing 4 T1.2 and 66c14 cell lines as well as the initial in vivo tumor progression studies. KT. was responsible for the transfection and selection of the luciferase-expressing 4 T1.2 and $66 \mathrm{c} 14$ cell lines. CB was responsible for the 4 T1.2 luc3 and 66c14 luc2 in vivo and ex vivo metastasis data along with the majority of all writing and figure compilations. JM was responsible for the 4 T1 luc2 in vivo metastasis study. CJ was the primary investigator responsible for directing all the work conducted in this manuscript. All authors read and approved the final manuscript.

\section{Authors' information}

Caleb Sutherland is currently a PhD graduate student in the Department of Cancer Biology at the University of Arizona in Tucson, AZ. Caleb conducted his research contributing to this manuscript while a McNair Scholar working in the laboratory for Dr. Cheryl Jorcyk at Boise State University. Ken Tawara, M.S. received his Master's degree in the laboratory of Dr. Cheryl Jorcyk in March of 2011 and performed the research pertaining to this manuscript while conducting his graduate studies. Dr. Celeste Bolin and Dr. Jim Moselhy are post-doctoral fellows in the laboratory of Dr. Cheryl Jorcyk. Dr. Cheryl Jorcyk is a full professor at Boise State University in the Department of Biological Sciences and collaborates with Dr. Robin Anderson who is head of the Metastasis Research Laboratory at the Peter MacCallum Cancer Centre in Melbourne, Australia.

\section{Acknowledgements}

Funding provided by ACS RSG-09-276-01-CSM, Susan G Komen for the Cure KG100513, NIH/NCRR P20RR016454, and NASA NNX10AN29A. Mice were maintained at the VA Medical Center, Boise, ID funded in part by NIH/NIAMS P30AR057235. Dr. Robin Anderson (Peter MacCallum Cancer Centre, Melbourne, Australia) generously provided the parental 4 T1.2 and 66c14 cell lines. Micro-CT analysis was generously performed by Anthony Hafez at the Biomedical Research Center, Boise State University (ISBOE Musculoskeletal Research Institute funding). This work was made possible in part by ImageVis3D software funded by the NIH/NCRR Center for Integrative Biomedical Computing, 2P41 RR0112553-12 and the DOE SciDAC Visualization and Analytics Center for Enabling Technologies, DEFC0206ER25781.

\section{Author details}

'Department of Biological Sciences, Boise State University, Boise, ID, USA. ${ }^{2}$ Present Address: Department of Cancer Biology, University of Arizona, Tucson, AZ, USA

Received: 25 January 2012 Accepted: 3 March 2012 Published: 17 April 2012

\section{References}

1. Weigelt B, Peterse $\mathrm{LL}$, van't Veer $\mathrm{L}$ : Breast cancer metastasis: Markers and models. Nat Rev Cancer 2005, 5(8):591-602.

2. Coleman RE: Skeletal complications of malignancy. Cancer 1997, 80 (8):1588-1594.

3. Coleman RE, Smith P, Rubens RD: Clinical course and prognostic factors following bone recurrence from breast cancer. Br J Cancer 1998, 77 (2):336-340

4. Kennecke $H$, Yerushalmi R, Woods $R$, Cheang MCU, Voduc D, Speers $\mathrm{CH}$, Nielsen TO, Gelmon K: Metastatic Behavior of Breast Cancer Subtypes. J Clin Oncol 2010, 28(20):3271-3277.

5. Ottewell PD, Coleman RE, Holen I: From genetic abnormality to metastases: murine models of breast cancer and their use in the development of anticancer therapies. Breast Cancer Res Treat 2006, 96 (2):101-113.

6. Donehower LA, Harvey M, Slagle BL, McArthur MJ, Montgomery CA, Butel JS, Bradley A: Mice deficient for $\mathrm{p} 53$ are developmentally normal but susceptible to spontaneous tumors. Nature 1992, 356(6366):215-221.

7. Fantozzi A, Christofori G: Mouse models of breast cancer metastasis. Breast Cancer Research 2006, 8(4):212-223.

8. Montel V, Mose ES, Tarin D: Tumor-stromal interactions reciprocally modulate gene expression patterns during carcinogenesis and metastasis. Int J Cancer 2006, 119(2):251-263.

9. Clarke R: Human breast cancer cell line xenografts as models of breast cancer. The immunobiologies of recipient mice and the characteristics of several tumorigenic cell lines. Breast Cancer Res Treat 1996, 39(1):69-86.

10. Lelekakis M, Moseley J, Martin T, Hards D, Williams E, Ho P, Lowen D, Javni J, Miller F, Slavin J, et al: A novel orthotopic model of breast cancer metastasis to bone. Clinical \& Experimental Metastasis 1999, 17(2):163-170

11. Eckhardt BL, Parker BS, van Laar RK, Restall CM, Natoli AL, Tavaria MD, Stanley KL, Sloan EK, Moseley JM, Anderson RL: Genomic analysis of a spontaneous model of breast cancer metastasis to bone reveals a role for the extracellular matrix. Mol Cancer Res 2005, 3(1):1-13.

12. Pulaski BA, Ostrand-Rosenberg S: Mouse $4 \mathrm{~T} 1$ Breast Tumor Model. In: Current Protocols in Immunology. edn.: John Wiley \& Sons, Inc.; 2001. 
13. Goldstein RH, Weinberg RA, Rosenblatt M: Of Mice and (Wo)men: Mouse Models of Breast Cancer Metastasis to Bone. J Bone Miner Res 2010, 25 (3):431-436.

14. Kim JB, Urban K, Cochran E, Lee S, Ang A, Rice B, Bata A, Campbell K, Coffee $R$, Gorodinsky $A$, et al: Non-invasive detection of a small number of bioluminescent cancer cells in vivo. PLoS One 2010, 5(2):e9364.

15. Cicek M, Iwaniec UT, Goblirsch MJ, Vrabel A, Ruan M, Clohisy DR, Turner RR, Oursler MJ: 2-Methoxyestradiol suppresses osteolytic breast cancer tumor progression in vivo. Cancer Res 2007, 67(21):10106-10111.

16. Zwolak P, Jasinski P, Terai K, Gallus NJ, Ericson ME, Clohisy DR, Dudek AZ: Addition of receptor tyrosine kinase inhibitor to radiation increases tumour control in an orthotopic murine model of breast cancer metastasis in bone. Eur J Cancer 2008, 44(16):2506-2517.

17. Goblirsch M, Zwolak P, Ramnaraine ML, Pan W, Lynch C, Alaei P, Clohisy DR: Novel cytosine deaminase fusion gene enhances the effect of radiation on breast cancer in bone by reducing tumor burden, osteolysis, and skeletal fracture. Clin Cancer Res 2006, 12(10):3168-3176.

doi:10.1186/1480-9222-14-6

Cite this article as: Bolin et al: Novel mouse mammary cell lines for in vivo bioluminescence imaging (BLI) of bone metastasis. Biological Procedures Online 2012 14:6

\section{Submit your next manuscript to BioMed Central and take full advantage of:}

- Convenient online submission

- Thorough peer review

- No space constraints or color figure charges

- Immediate publication on acceptance

- Inclusion in PubMed, CAS, Scopus and Google Scholar

- Research which is freely available for redistribution 